\title{
Analysis of Thoracoabdominal Motion during Incremental Work Cycle Exercise
}

\author{
AKIRA TAMAKI, MS, RPT, RCE ${ }^{1,4)}$, ARISA KITADA, RPT ${ }^{2)}$, YoshIMI MATSUO, RPT ${ }^{3)}$, \\ SATORU INOUE, RPT ${ }^{3)}$, JUNZO TSUJITA, MS ${ }^{4}$, SEIKI HORI, MD ${ }^{4}$ \\ ${ }^{1)}$ Department of Physical Therapy, Osaka Prefectural College of Health Sciences: 3-7-30, \\ Habikino, Osaka 583-8555, Japan. TEL +81-729-50-2111 FAX +81-729-50-2130 \\ ${ }^{2)}$ Department of Rehabilitation, Gracia Hospital \\ ${ }^{3)}$ Rehabilitation Unit, Osaka University Hospital \\ ${ }^{4)}$ Department of Physiology, Hyogo College of Medicine
}

\begin{abstract}
We measured thoracoabdominal motion by analyzing changes of rib cage (RC) and abdomen (ABD) motion during cycle ergometry using respiratory inductive plethysmography in eight healthy subjects (8 males). Cycle exercise was performed at three incremental work rates of 60,90 , and $120 \mathrm{w}$ for five minutes each and tidal volume (TV) was continuously measured with a respiratory flowmeter on a breath-by-breath basis. It was found that RC motion increased, but ABD motion did not always increase, during incremental work cycle exercise RC motion contributed more to the increase of TV than ABD motion. These results suggest that TV increase was produced more by RC motion than by ABD motion.
\end{abstract} Key words: Thoracoabdominal motion, Respiratory inductive plethysmography, Incremental exercise.

(This article was submitted Apr. 25, 2000, and was accepted Sep. 20, 2000)

\section{INTRODUCTION}

Breathing pattern is one of the most important components of pulmonary evaluation. There are many reports ${ }^{1-7)}$ about breathing pattern or thoracoabdominal motion at rest.

For example, Verschakelen and Demedts ${ }^{1)}$ found that under most conditions the rib cage (RC) motion predominated over the abdominal (ABD) motion except during quiet breathing in the supine position, and Sharp et al. ${ }^{2)}$ reported that rapid respiratory maneuvers were accomplished mostly through rib cage displacement. However, they did not measure thoracoabdominal motion during exercise.

So far, there have been few studies ${ }^{8-10)}$ during exercise because it is difficult to measure thoracoabdominal motion.

The purpose of this study was to assess characteristics of thoracoabdominal motion during exercise by analyzing changes of $\mathrm{RC}$ and $\mathrm{ABD}$ motion during cycle ergometry using respiratory inductive plethysmography.

\section{SUBJECTS}

Eight healthy subjects ( 8 males) participated in this study after giving their written informed consent. Their mean age was $24.5 \pm 3.5$ years old, mean height was $175.9 \pm 8.0 \mathrm{~cm}$, and mean weight was $64.8 \pm 5.9 \mathrm{~kg}$.

All subjects had no history of lung disease and had no orthopedical or neurological disorders.

\section{METHODS}

Cycle exercise was performed on the same day in a randomized order for each subject. Following one minute warm-up of unloaded cycling, after taking enough rest in a sitting position, subjects pedaled at three incremental work rates of 60,90 , and $120 \mathrm{~W}$ 


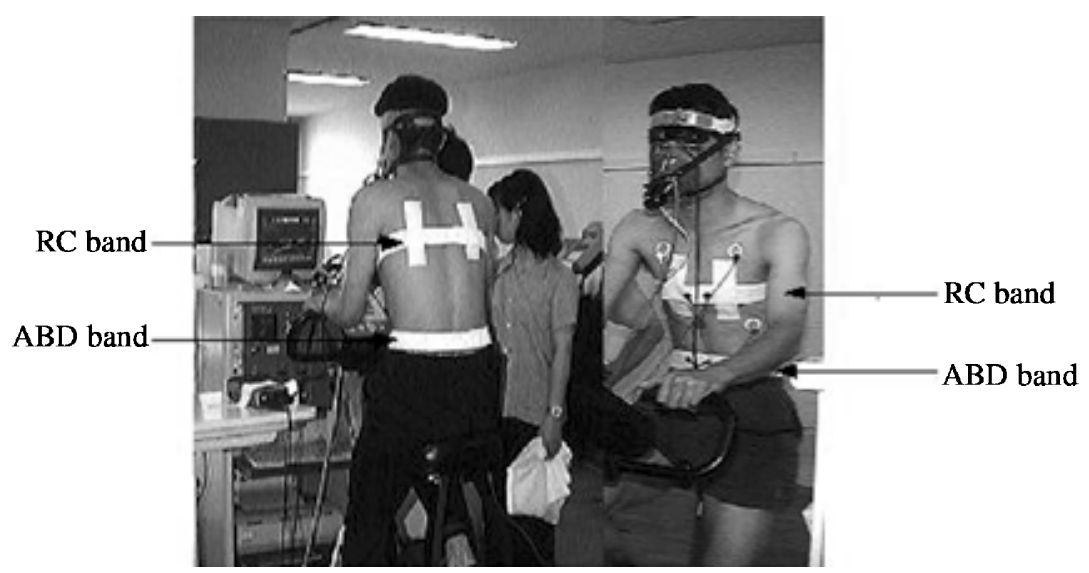

Fig. 1. Measurement of Thoracoabdominal motion. The change of thoracoabdominal motion was measured as a peak-to-peak value every breath during the last minute of each work (steady-state phase), and averaged $(\triangle \mathrm{RC}$ and $\triangle \mathrm{ABD})$.

for five minutes each. During cycle exercise, tidal volume (TV) was continuously measured with a respiratory flowmeter (MINTO AE-280) on a breath-by-breath basis, and averaged to obtain mean values every 30 seconds.

Thoracoabdominal motions were measured using a respiratory inductive plethysmograph (Sensor Medics-Respitrace Plus, San Diego, CA).

Respiratory inductive plethysmography has been widely used for studies of ventilation during sleep and can measure breathing without direct mechanical coupling to the mouth and nose. With this device, pulmonary ventilation is defined as the change in the lung volume, which is calculated by measuring changes in the circumference of the chest and abdomen.

The change in circumference was determined by changes in the electrical inductance of the band fixed around the rib cage and umbilicus in each subject (Fig. 1). We defined $100 \%$ VT to be the average volume of chest and abdominal motion during the initial 5-minutes calibration period at rest in the sitting position on a bicycle ergometer. The accuracy of this device has been previously reported ${ }^{11)}$.

The analog data of TV and thoracoabdominal motion were input into a personal computer, and synchronized with a software program (Biopack System Inc, San Diego, CA) as shown in Fig. 2.

The change of thoracoabdominal motion was measured as a peak-to-peak value every breath during the last minute of each work (steady-state

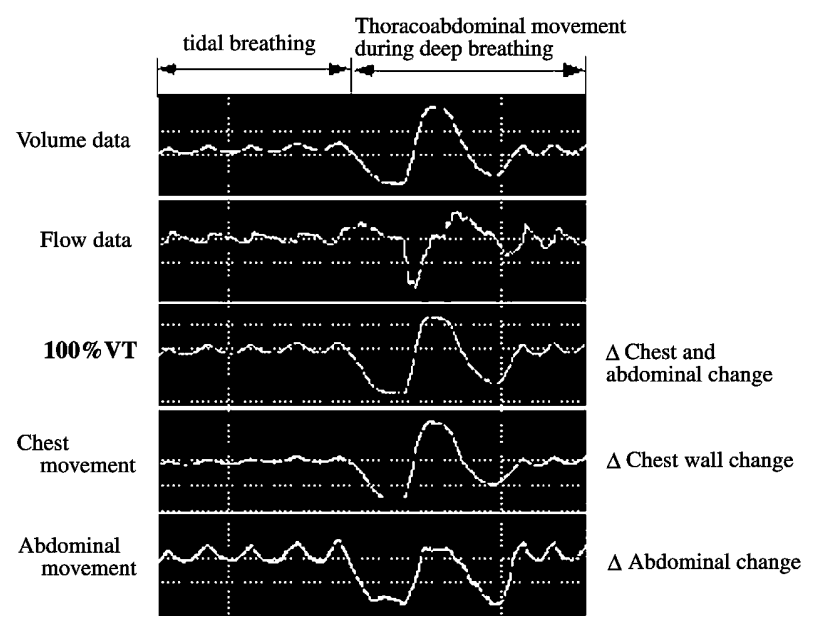

Fig. 2. Tidal breathing and Thoracoabdominal motion. The analog data of TV and Thoracoabdominal motion were input into a personal computer and synchronized.

phase), and averaged.

For statistical analysis, TV and the change of RC and ABD motions during incremental work cycle exercise were subjected to multiple regression analysis. Statistical significance was defined as $\mathrm{p}<0.05$.

\section{RESULTS}

\section{The change of $R C$ and $A B D$ motion}

The change of RC and ABD motion during 

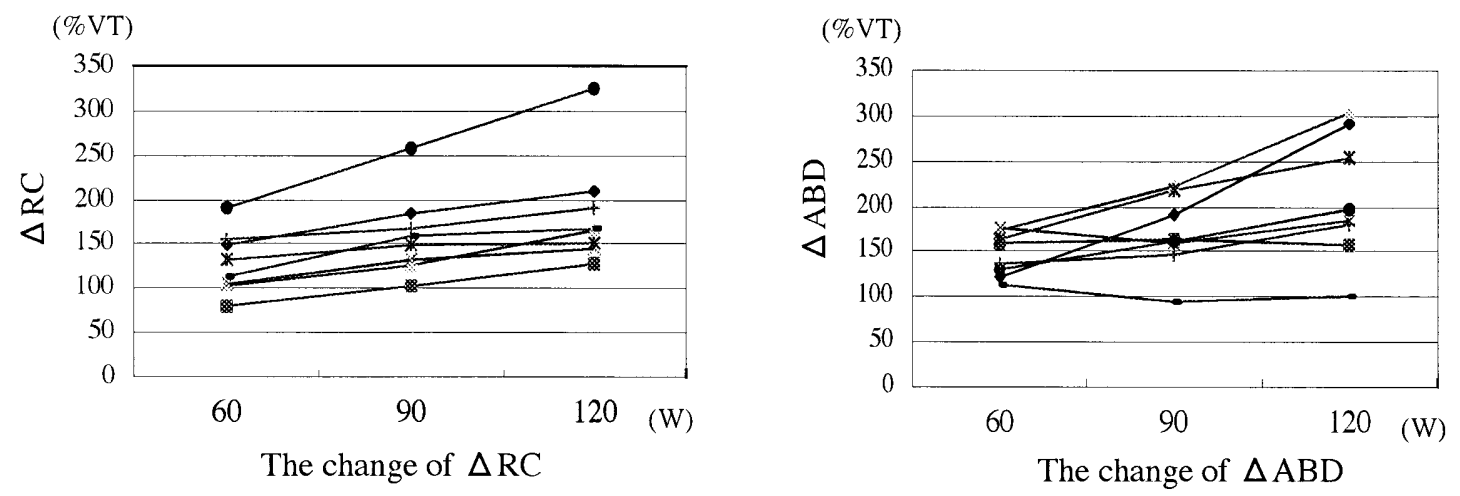

Fig. 3. The change of $\triangle \mathrm{RC}$ and $\triangle \mathrm{ABD}$ during exercise. Left: As work load increased, $\mathrm{RC}$ motion increased for all subjects. However, the slope of the RC motion response was irregular among subjects. Right: ABD motion did not increase in all subjects during exercise. Subjects were not told that the slopes of ABD motion responses were irregular.

Table 1. The change in the ratio of $\triangle \mathrm{RC}$ to $\triangle \mathrm{ABD}$ during exercise

\begin{tabular}{lccc}
\hline & $60 \mathrm{~W}$ & $90 \mathrm{~W}$ & $120 \mathrm{~W}$ \\
\hline $\mathrm{A}$ & 1.23 & 0.97 & 0.6 \\
$\mathrm{~B}$ & 0.50 & 0.63 & 0.8 \\
$\mathrm{C}$ & 0.59 & 0.56 & 0.54 \\
$\mathrm{D}$ & 0.58 & 0.84 & 0.77 \\
$\mathrm{E}$ & 0.80 & 0.68 & 0.59 \\
$\mathrm{~F}$ & 1.42 & 1.58 & 1.63 \\
$\mathrm{G}$ & 1.14 & 1.15 & 1.05 \\
$\mathrm{H}$ & 1.24 & 1.67 & 1.65 \\
\hline & & \multicolumn{3}{c}{$(\Delta \mathrm{RC} / \Delta \mathrm{ABD})$}
\end{tabular}

The change pattern of RC motion to $\mathrm{ABD}$ motion (RC/ABD) is irregular. For the change from beginning of exercise to end of $120 \mathrm{~W}$, subjects were classified into two groups: 4 persons increased RC motion more than ABD motion, and 4 persons increased $\mathrm{ABD}$ motion more than $\mathrm{RC}$ motion.

exercise is shown in Fig. 3.

As work load increased, RC motion increased for all subjects, however, the slope of the RC motion response was irregular among the subjects.

Not all subjects showed an increase in ABD during exercise. So, subjects were classified in two groups: the increasing tendency group which included six persons, and the decreasing tendency group, with two persons.

Each group did not indicate that the slope of ABD motion responses were irregular.

The rate of change of RC motion to ABD motion (RC/ABD) was irregular (Table 1). For the change rate from beginning of exercise to the end of $120 \mathrm{~W}$, subjects were classified in two groups: four persons increased $\mathrm{RC}$ motion more than $\mathrm{ABD}$, and four persons increased $\mathrm{ABD}$ motion more than RC.

\section{The change of ventilation}

As the work load increased, TV did not increase in all subjects.

With regard to the relation of $\mathrm{TV}$ to thoracoabdominal motion, TV correlated more with RC motion (0.87) than with ABD motion (0.69) (Fig. 4).

For all subjects, the combined TV, RC and ABD relationship during exercise was described by the equation $\mathrm{TV}=312.99+0.793(\mathrm{RC})+0.112(\mathrm{ABD})$; also, $\mathrm{RC}$ motion contributed to a greater degree to TV.

\section{DISCUSSION}

The purpose of this study was to assess the changes of RC and ABD motion during incremental work cycle exercise for healthy subjects.

As the work load increased, all subjects showed an increase in RC motion, however, ABD motion was irregular.

We concluded that ABD motion did not increase because of obstruction of the ABD motion by something. Muscle activity of trunk muscles during cycle exercise was expected.

Grimby et al. ${ }^{8)}$ reported that there was a possibility that abdominal muscle contraction could effect $\mathrm{ABD}$ motion. But there are no reports of a 

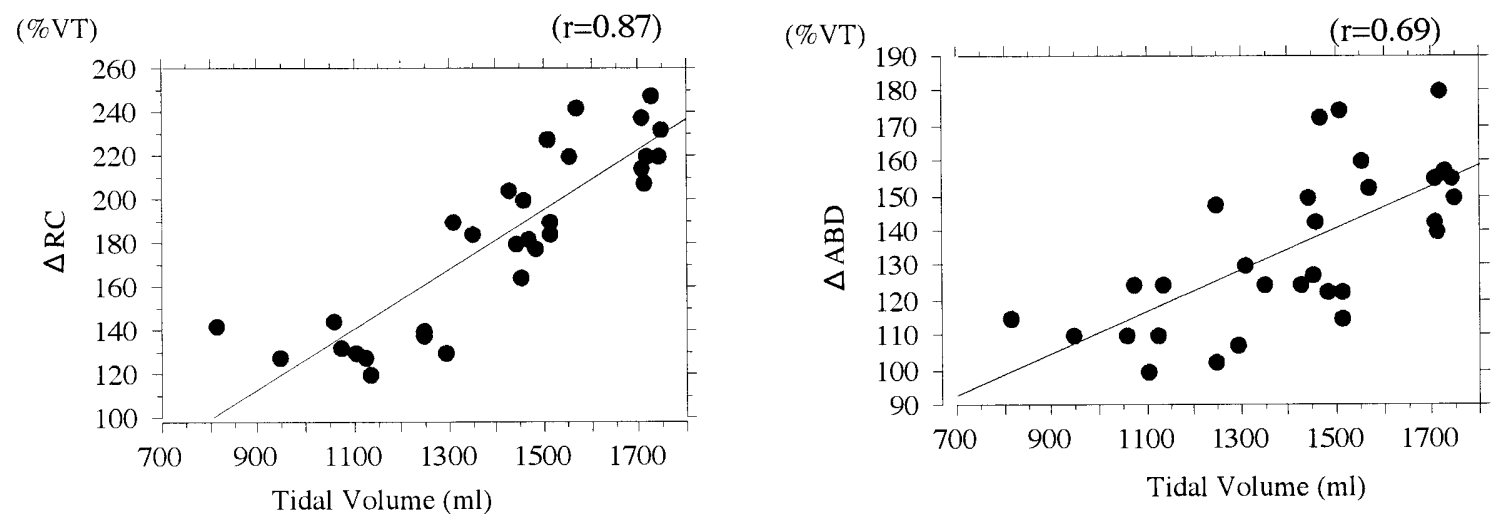

Fig. 4. Correlation between $\mathrm{TV}$ and $\triangle \mathrm{RC}$ and $\triangle \mathrm{ABD}$. In the relation of $\mathrm{TV}$ to thoracoabdominal motion, $\mathrm{TV}$ correlated more with RC motion than with $\mathrm{ABD}$ motion; also, RC motion contributed to a greater degree to TV.

direct relation between thoracoabdominal motion and trunk muscle contraction during exercise, so further studies are necessary.

The present results indicated that subjects could be classified into a greater RC increase group and a greater $\mathrm{ABD}$ increase group; the change rate of $\mathrm{RC}$ and $A B D$ motion was irregular. Our results are in agreement with the account given by Komuro et al. ${ }^{9)}$.

With regard to the relation of $\mathrm{TV}$ to thoracoabdominal motion, it was indicated that the increase of TV during exercise depended more on RC motion than on ABD motion; also RC contributed to a greater degree to TV. Among the subjects, though, TV increased together with decreased ABD motions in some subjects.

In general, TV increased by the increase of RC and ABD motion, but all subjects did not show a relative increase of ABD motion to TV. That is to say, it was indicated that the increase of TV during exercise depended more on RC motion than on ABD motion.

Clinically, ABD motion has tended to receive more attention as a breathing exercise for the patients with pulmonary diseases. But, we thought that it would be easier to get the needed ventilation volume by a gain in the reserved motion of the rib cage by training (for example, to improve the mobility of the upper thorax).

\section{CONCLUSION}

We assessed the change in values of thoracoabdominal motion during cycle exercise in healthy subjects. RC motion increased, but ABD motion did not always increase during incremental work cycle exercise in healthy subjects. RC motion contributed more to the increase in TV than ABD motion. We found that the change rates of thoracoabdominal motion during cycle exercise were irregular.

\section{REFERENCES}

1) Verschakelen JA, Demedts MG: Normal thoracoabdominal motions: influence of sex, age, posture, and breath size. 1995, Am J Respir Crit Care Med 151: 399-405.

2) Sharp JT, Goldberg NB, Druz WS, et al.: Relative contributions of rib cage and abdomen to breathing in normal subjects. 1975, J Appl Physiol 39 (4): 608-618.

3) Sharp JT, Goldberg NB, Druz WS, et al.: Thoracoabdominal motion in chronic obstructive pulmonary disease. 1977, Am Rev Respir Dis 115 (1): 47-56.

4) Vellody VP, Nassery M, Druz WS, et al.: Effects of body position change on thoracoabdominal motion. 1978, J Appl Physiol 45 (4): 581-589.

5) Faithfull D, Jones JG, Jordan C: Measurement of the relative contributions of rib cage and abdomen/ diaphragm to tidal breathing in man. 1979, Br J Anaesth 51 (5): 391-398.

6) Estenne M, Yernault JT, Troyer A: Rib cage and diaphragm-abdomen compliance in humans: effects of age and posture. 1985, J Appl Physiol 59 (6): 18421848.

7) Siafakas NM, Morris AJ, Green M: Thoracoabdominal mechanics during relaxed and forced vital capacity. 1979, J Appl Physiol 47 (1): 38-42.

8) Grimby G, Bunn J, Mead J: Relative contribution of rib cage and abdomen to ventilation during exercise. 1968, 
J Appl Physiol 24: 159-166.

9) Komuro $T$, Mase $K$, Imura $S$, et al.: Change of breathing pattern (Frequency-tidal volume relation) and rib cage-abdominal motion during exercise in normal subjects. 1993, Regaku ryouhougaku 21 (8): 549-552 (in Japanese).

10) Wells JA, Smyth RJ, Rebuck AS: Thoracoabdominal motion in response to treadmill and cycle exercise. 1986, Am Rev Respir Dis 134 (6): 1125-1128.

11) Caretti, DM, Pullen PV, Premo LA et al.: Reliability of respiratory inductive plethysmography for measuring tidal volume during exercise. 1994, Am Ind Hyg Assoc J 55: 918-923. 